\title{
Evaluación psicopatológica en dependientes al Alcohol, Heroína y Cocaína mediante el Brief Symptom Inventory
}

\author{
Sánchez-Hervás, E.; Tomás Gradolí, V.; Morales Gallús, E. \\ Unidad de Conductas Aditivas, Área 9. Catarroja. \\ Conselleria de Sanitat. Valencia \\ Enviar correspondencia a: \\ Emilio Sánchez Hervás. Unidad de Conductas Adictivas-Centro de Salud de Catarroja. Avd. Rambleta s/n 46470 CATARROJA - Valencia.
}

\section{RESUMEN}

Objetivos: analizar la presencia de psicopatología en los usuarios de una Unidad de Conductas Adictivas mixta (tratamiento de la adicción a drogas legales e ilegales). Comprobar si los pacientes presentan más psicopatología que un grupo de sujetos sin problemas por uso de drogas.

Sujetos y Método: 216 pacientes distribuidos en cuatro grupos: tres grupos formados por pacientes en tratamiento (diagnóstico de dependencia a alcohol $n=43$, diagnóstico de dependencia a heroína $n=65$, diagnóstico de dependencia a cocaína $n=42$ ), más un grupo control $(n=66)$ formado por sujetos sin problemas de abuso de drogas. A todos los sujetos se les administró el Brief Symptom Inventory (Derogatis, 1975), para evaluar la presencia de psicopatología. Se realizan análisis de frecuencias y medidas de tendencia central, contraste de diferencias mediante análisis de varianza y comparaciones múltiples con la prueba de Schefeé.

Resultados y Discusión: los grupos formados por pacientes presentan gran variedad de síntomas psicopatológicos. Las puntuaciones del grupo control son significativamente más bajas que las obtenidas por los grupos de pacientes en todos los indicadores de psicopatología. De los grupos de pacientes, es el grupo de alcohólicos el que presenta un mayor número de indicadores con significación estadística cuando se comparan al grupo control No aparecen diferencias cuando se comparan entre sí los grupos de pacientes. Destacan las características sociodemográficas del grupo alcohol (mayor edad media y mayor número de años de consumo que el resto de grupos de pacientes), es posible que ello influya en las puntuaciones de psicopatología de este grupo. El grupo de pacientes de cocaína es el que obtiene puntuaciones mayores en el índice global de severidad y en el total de síntomas. Las puntuaciones en los índices globales de psicopatología podrían indicar un nivel de malestar psicológico que interfiriera en el mantenimiento de la abstinencia. Finalmente se señala la importancia de evaluar la psicopatología en los pacientes que acuden a tratamiento.

Palabras clave: psicopatología, alcoholismo, dependencia a heroína, dependencia a cocaína.

\section{ABSTRACT}

Objectives: To analyse the presence of psychophatology in users of a mixed Addictive Behaviours Unity (treatment of legal and illegal drugs addiction). To verify in-patients show more psychophatology than one group without problems for use of drugs.

Subjects and Method: 216 patients distributed in four groups: three groups composed by patients in treatment (alcohol dependence $n=43$, heroin dependence $n=65$, cocaine dependence $n=42)$, plus a control group ( $n=66)$ composed by subjects without problems of drugs abuse. The Brief Symptom Inventory (Derogatis, 1975) was administered to all patients in order to evaluate the presence of psychophatology. Analysis of frequencies and measurement central tendency of contrast of differences through analysis of variance and multiple comparisons with Schefeé test has been realized.

Results and Discussion: The groups composed by patients show a big variety of psychophatology symptoms. The punctuation's of control group are significantly more lower than the punctuation's obtained by the patient groups. From among patient groups, alcohol group shows a bigger number of indicators with statistic significance on comparing with control group. Differences don't show on comparing the patient groups.

Emphasize the sociodemographic characteristics of the group alcohol (greater mean and greater age number of consumption years that the rest of groups of patients), it is possible that this influence the punctuations of psychophatology of this group. The group of cocaine patients is the one which obtains greater punctuations in the global severity index and in the symptoms total. The punctuations in the global indices of psychophatology could indicate a psychological discomfort level that to inder in the maintenance of the abstinence. Finally the implications of these results are discussed and we mark the importance of evaluating the psychophatology in-patients who demand treatment.

Key words: psychophatology, alcoholism, heroin dependence and cocaine dependence.

\section{INTRODUCCIÓN}

n número importante de los pacientes que son atendidos en los centros de atención a drogodependientes presentan criterios diagnósticos de uno o varios trastornos de personalidad así cómo de otros trastornos psiquiátricos (Abbot, 1994). La fun- cionalidad del consumo de drogas en este tipo de pacientes puede ser distinta a la de aquellos pacientes que no presentar indicadores de psicopatología y, por tanto, la intervención que se realice debe de contemplar tal extremo. Aquellos pacientes que no presentan indicadores de psicopatología añadidos a su comportamiento adictivo, mostraran a los pocos días de iniciar el tratamiento una mayor estabilidad, un 
descenso en sus niveles de ansiedad y una mejora de sus relaciones interpersonales. Por el contrario, los pacientes que presenten síntomas psicopatológicos y, quizás otros trastornos psiquiátricos, tendrán con frecuencia dificultades para mantener la abstinencia a largo plazo, recaídas en el consumo y realizarán numerosos intentos de deshabituación en distintos programas de tratamiento.

Se pueden encontrar en la bibliografía distintos estudios que ponen de manifiesto la relación existente entre el abuso de drogas y psicopatología. Jellinek (1960) ya señaló hace bastantes años que el deseo de bebida en los alcohólicos era fruto de una dimensión obsesiva o impulsiva. Se ha mencionado en numerosos trabajos la asociación entre depresión y alcoholismo (Richam y col, 1980; Schukit, 1985; Van Hasselt, 1993), entre ansiedad y alcoholismo (Neff, 1993; Sayette, 1993), y alcoholismo con otros indicadores de psicopatología como la hipocondría y la desviación psicopática (Ochando y col, 1999), trastornos de personalidad (De Vicente y col, 1999), y otras complicaciones somáticas (Cuadrado, 1998).

Con respecto a adictos a opiáceos también se ha mencionado la elevada prevalencia de distintos trastornos que presentan estos pacientes (Rousanville y col, 1982; Khantzian y Treecee, 1985; Miller, 1995, 1996, Wojnar, 1997). Se ha evidenciado que muchos de los pacientes cumplen criterios para diagnosticar trastornos de personalidad (Lombardi, 1993; Goldstein, 1996; Calsyn y col, 1996; Blaszczynsky y col, 1997; San Narciso y col, 1998; Gutierrrez y col, 1998; Sánchez-Hervás y col, 1999).

También se encuentran referencias a propósito de la psicopatología en adictos a cocaína. Numeros estudios señalan una asociación entre la adicción a cocaína con trastornos del estado de ánimo; (Gawin y Kleber, 1986; Nunes y col, 1986; Sorel, 1990; Gold, 1993); trastornos de ansiedad (Nunes y col, 1986, Gold, 1989; Post y col, 1992); trastornos de personalidad (Fielman y col, 1995; Yates y col, 1995); sintomatología y trastornos psicóticos (Siegel, 1982; Catton y col, 1989; Havassy y Arns, 1998).

Parece pues apropiado, dada la evidencia de la existencia de psicopatología en los pacientes que demandan tratamiento por abuso de drogas, evaluar la presencia de indicadores que puedan orientar en el diagnóstico y planificación de la intervención. El trabajo que se presenta a continuación pretende situarse en esta línea.

\section{MATERIAL Y MÉTODO}

\section{Objetivos}

Analizar la psicopatología y las características sociodemográficas de un grupo de pacientes en tratamiento en la Unidad de Conductas Adictivas Area 9.
Catarroja (Valencia). Comprobar si los pacientes atendidos presentan más psicopatología que un grupo de sujetos sin diagnóstico de dependencia a drogas.

\section{Muestra}

La muestra la componen 216 sujetos divididos en cuatro grupos: tres grupos corresponden a pacientes de la UCA que demandaron tratamiento durante el año 1999 y se distribuyen en función del diagnóstico realizado: Grupo A, dependencia a alcohol $(n=43)$; grupo $B$, dependencia a heroína $(n=65)$ y, grupo $C$, dependencia a cocaína $(n=42)$. El cuarto grupo está formado por sujetos que no presentan dependencia a drogas y forman el grupo de control $(n=66)$.

Criterios de inclusión: dependencia a alcohol (grupo A), dependencia a heroína (grupo B) y dependencia a cocaína (grupo C).

Criterios de exclusión: consumo de la sustancia de abuso en las últimas cuatro semanas. Presentar diagnóstico de dependencia a más de una sustancia.

\section{Diseño}

Estudio transversal-retrospectivo

\section{Instrumento}

1.Entrevista estructurada: utilizada para todos los pacientes del servicio. Dicha entrevista recoge datos sociodemográficos, legales, laborales, orgánicos, historia toxicológica, tratamientos previos, indicadores psicopatológicos y demanda. En el trabajo se utilizan las variables edad, sexo, situación laboral y nivel de estudios.

2.Brief Symptom Inventory (BSI) de Derogatis (1975): instrumento de screening; forma abreviada del SCL-90 del que también es autor Derogatis y, del que posteriormente apareció una versión revisada, el SCL90-R (Derogatis, 1983). El BSI tiene una buena correlacion con el SCL-90, por encima de 0.90 en todas las dimesiones (Derogatis y Cleary, 1977), y además es mucho más ágil en su administración (53 ítems), lo que facilita su administración en usuarios de drogas. Consta de 53 ítems, que el usuario valora en una escala tipo Likert de 5 puntos (desde nada=0, hasta 4=mucho), que expresa en que intensidad se ha sentido molesto el usuario por la descripción del ítem en los últimos 7 días. Las puntuaciones medias en cada dimensión se obtienen de las medias correspondientes a las respuestas de los ítems que componen cada dimensión, y las puntuaciones en los índices globales se obtienen a partir de las puntuaciones en todas las dimensiones.

El BSI incluye (al igual que el SCL-90) las siguientes dimensiones sintomáticas: somatización, obsesióncompulsión, sensibilidad interpersonal, depresión, 
ansiedad, hostilidad, ansiedad fóbica, ideación paranoide y, psicoticismo. Además de estas nueve dimensiones, se incluyen tres índices globales que describen la intensidad de la psicopatología del encuestado: índice de severidad global, índice de malestar y, total de síntomas positivos. El índice de severidad global (GSI) proporciona un indicador sensible de la respuesta del nivel de estrés experimentado por el paciente. Combina la información del número de síntomas presentes y la intensidad del distress. El índice de malestar (PSDI) es una medida de la intensidad del nivel de distress esperimentado en función del número de síntomas, informando sobre el estilo de respuesta de distress. Finalmente, el indicador total de síntomas positivos (PST) revela el número de síntomas que el paciente afirma experimentar y, se utiliza conjuntamente con los otros indicadores globales comunicando la amplitud del distress emocional del individuo.

\section{Variables}

Variables sociodemográficas: edad, sexo, nivel de estudios, situación laboral. Obtenidas de las historias clínicas de los pacientes.

Variables dependientes: dimensiones del BSI (somatización, ansiedad, ansiedad fóbica, obsesivocompulsivo, depresión, hostilidad, sensibilidad interpersonal, ideación paranoide y psicoticismo); índices globales del BSI (GSI, PSDI, PST)

\section{Análisis de datos}

Para la descripción de la muestra, y para la estimación de las puntuaciones en el instrumento de evaluación se han realizado análisis de frecuencias y medidas de tendencia central. Para el contraste de diferencias se utilizó la prueba ji-cuadrado, se realizó un análisis de varianza y, para la comparación de grupos se utilizó la prueba de Scheffé. En la interpretación de resultados se eligió un nivel de significación estadística de $\mathrm{p}<0.05$, para un intervalo de confianza del $95 \%$. Todos los análisis se realizaron con el paquete estadístico SPSS 8.0 para Windows.

\section{RESULTADOS}

En la tabla 1 se muestran los descriptivos de la muestra. La edad media expresada en años en todos los grupos de estudio es similar (grupo cocaína 25,5; grupo heroína 27,8; grupo control 27,8), destaca la media del grupo alcohol $(40,3)$. Al comparar los grupos aparecen diferencias significativas entre el grupo alcohol y el resto de grupos. También destaca la media de años de consumo en el grupo alcohol $(22,5)$, siendo la más baja la del grupo cocaína $(5,2)$ y algo más elevada la del grupo heroína $(8,1)$. Las diferencias son estadís- ticamente significativas al comparar el grupo alcohol con los grupos cocaína y heroína.

La distribución por sexos es similar para los grupos cocaína y alcohol (90,5\% vs 90,7\% de varones), el porcentaje es menor en el caso del grupo heroína (75,4\% varones). Aparecen diferencias significativas de los grupos alcohol y cocaína frente a los grupos heroína y control. Respecto al nivel de estudios, la mayor parte de la muestra cursó solamente estudios primarios $(90,8 \%$ grupo heroína, 90,5\% grupo cocaína). En el grupo alcohol aparece un mayor porcentaje de pacientes con estudios secundarios respecto a los demás grupos (20,9\%). Existen diferencias significativas en las comparaciones de los grupos control y alcohol respecto a los grupos heroína y cocaína. Finalmente, la situación laboral difiere según el grupo de estudio. Los pacientes del grupo cocaína presentan un mayor porcentaje de sujetos activos laboralmente $(73,8 \%)$, frente a un $44,2 \%$ del grupo alcohol y un $32,3 \%$ del grupo heroína. El grupo cocaína presenta diferencias significativas al compararse a los grupos alcohol, heroína y control.

TABLA 1. Descriptivos muestra

\begin{tabular}{|c|c|c|c|c|c|c|}
\hline & & $\begin{array}{c}\text { Grupo } \\
\text { Control } \\
n=66\end{array}$ & $\begin{array}{c}\text { Grupo } \\
\text { Alcohol } \\
n=43\end{array}$ & $\begin{array}{c}\text { Grupo } \\
\text { Heroína } \\
n=65\end{array}$ & $\begin{array}{c}\text { Grupo } \\
\text { Cocaína } \\
n=42\end{array}$ & $\begin{array}{c}\text { ANOVA } \\
\chi^{2}\end{array}$ \\
\hline EDAD & $\begin{array}{c}\text { Media } \\
\text { SD } \\
\text { Rango }\end{array}$ & $\begin{array}{c}25,5 \\
6,45 \\
18-48\end{array}$ & $\begin{array}{c}40,3 \\
11,1 \\
28-60\end{array}$ & $\begin{array}{c}27,8 \\
5,15 \\
18-45\end{array}$ & $\begin{array}{c}26,5 \\
6,72 \\
18-43\end{array}$ & $\begin{array}{c}0,000^{a, b, c} \\
\$\end{array}$ \\
\hline \multirow[t]{2}{*}{$\begin{array}{c}\text { AÑOS DE } \\
\text { CONSUMO }\end{array}$} & $\begin{array}{l}\text { Media } \\
\text { SD } \\
\text { Rango }\end{array}$ & - & $\begin{array}{c}22,5 \\
10,8 \\
10-42\end{array}$ & $\begin{array}{c}8,1 \\
5,1 \\
1-18\end{array}$ & $\begin{array}{c}5,2 \\
3,81 \\
1-13\end{array}$ & $\begin{array}{c}0,000^{d} \\
\$\end{array}$ \\
\hline & & $\%$ & $\%$ & $\%$ & $\%$ & \\
\hline \multirow[t]{2}{*}{ SEXO } & Hombre & 75,8 & 90,7 & 75,4 & 90,5 & $0,000^{e, \&}$ \\
\hline & Mujer & 24,2 & 9,3 & 24,6 & 9,5 & \\
\hline \multirow[t]{2}{*}{ ESTUDIOS } & Primarios & 78,8 & 79,1 & 90,8 & 90,5 & $0,000^{\dagger, 8}$ \\
\hline & Secundarios & 21,2 & 20,9 & 9,2 & 9,5 & \\
\hline \multirow{2}{*}{$\begin{array}{l}\text { SITUACIÓN } \\
\text { LABORAL }\end{array}$} & En paro & 66,6 & 55,8 & 67,7 & 26,3 & $0,000^{9, \&}$ \\
\hline & Activo & 33,4 & 44,2 & 32,3 & 73,8 & \\
\hline
\end{tabular}

\$-Valores medios y desviación estándar de las características sociodemográficas. Comparación entre medias mediante ANOVA. Comparación entre grupos mediante la prueba de Schefée. Diferencias con $p<0.05$. \&- Frecuencias comparación con ji-cuadrado.

${ }^{\mathrm{a}} \mathrm{Gr}$. Control vs Gr. Alcohol, ${ }^{\mathrm{b}} \mathrm{Gr}$. Control vs Gr Heroína, 'Gr. Alcohol vs Gr. Cocaína.

${ }^{\mathrm{d} G r}$. Alcohol vs Gr. Heroína y Cocaína, ${ }^{\mathrm{e}} \mathrm{Gr}$. Cocaína y Alcohol vs Gr. Heroína y Gr. Control, ${ }^{\dagger} \mathrm{Gr}$. Control y Alcohol vs Gr. Heroína y Gr. Cocaína, ${ }^{9}$ Gr. Cocaína vs Gr. Alcohol, Gr. Heroína y Gr. Control.

En la tabla 2 se muetran las puntuaciones de todos los grupos de estudio en las dimensiones e índices globales del BSI, la comparación de medias mediante análisis de varianza (ANOVA) y, la comparación entre grupos realizada con la prueba de Scheffé. El grupo control es el que obtiene las puntuaciones más bajas en todas las dimensiones e índices. Las puntuaciones más elevadas del grupo alcohol corresponden a las dimensiones obsesivo-compulsivo, ideación paranoide y depresión. Las más bajas corresponden a las dimensiones ansiedad fóbica, somatización y psicoticismo. Las puntuaciones más elevadas en el grupo heroína 
aparecen en las dimensiones obsesivo-compulsivo e ideación paranoide y, las más bajas en las dimensiones ansiedad fóbica y psicoticismo. Las puntuaciones más elevadas en el grupo cocaína corresponden a las dimensiones ideación paranoide, obsesivo-compulsivo, depresión y hostilidad. Las más bajas corresponden a las dimensiones ansiedad fóbica y somatización. Las puntuaciones más bajas del grupo control corresponden a las dimensiones ansiedad fóbica y somatización. Las puntuaciones más elevadas aparecen en las dimensiones obsesivo-compulsivo e ideación paranoide.

Respecto a los índices globales el grupo cocaína es el que más elevado puntúa en el índice de severidad global (GSI) y en el total de síntomas (PST). El grupo heroína puntúa más bajo en el índice de malestar (PSDI).

El resultado del contraste de medias realizado muestra la existencia de diferencias significativas en todas las dimensiones e índices del instrumento de evaluación a excepción de la dimensión obsesivocompulsivo ( $p=0.687$ ). Los resultados de las diferentes dimensiones son: somatización, depresión, psicoticismo, ideación paranoide, GSI y PST ( $p=0.000)$, sensibilidad interpersonal $(p=0.001)$, ansiedad $(p=0.002)$, ansiedad fóbica $(p=0.012)$, PSDI $(p=0.024)$ y hostilidad $(p=0.036)$.

Para comprobar el sentido de las diferencias encontradas, se procedió a una comparación múltiple de los grupos, utilizando la prueba de Scheffée. Los resultados muestran la existencia de diferencias cuando se comparan los grupos de pacientes, (alcohol, heroína y cocaína), con el grupo control, pero no aparecen diferencias cuando se comparan grupos de pacientes entre sí. El grupo alcohol es el que presenta un mayor número de dimensiones e índices estadísticamente significativos al compararse con el grupo control, somatización, sensibilidad interpersonal, depresión, ansiedad, ansiedad fóbica, ideación paranoide, psicoticismo, GSI, PSDI y PST.

Al comparar el grupo heroína con el grupo control, aparecen diferencias significativas en las dimensiones somatización, sensibilidad interpersonal, depresión, ansiedad, ideación paranoide, GSI y PST.

El grupo cocaína presenta diferencias significativas con el grupo control en las dimensiones sensibilidad interpersonal, depresión, hostilidad, ideación paranoide, psicoticismo, GSI y PST.

\section{DISCUSIÓN}

Los resultados de nuestro estudio se sitúan en la línea de hallazgos en otros trabajos. Se han obtenido puntuaciones más altas en todos los indicadores de psicopatología en los grupos de pacientes que en el grupo control. Estas puntuaciones muestran diferen-
TABLA 2. Puntuaciones BSI

\begin{tabular}{|c|c|c|c|c|c|c|}
\hline & $\begin{array}{c}\text { Grupo } \\
\text { Control } \\
\mathbf{n}=\mathbf{6 6}\end{array}$ & $\begin{array}{c}\text { Grupo } \\
\text { Alcohol } \\
\mathbf{n}=\mathbf{4 3}\end{array}$ & $\begin{array}{c}\text { Grupo } \\
\text { Heroína } \\
\mathbf{n = 6 5}\end{array}$ & $\begin{array}{c}\text { Grupo } \\
\text { Cocaína } \\
\mathbf{n = 4 2}\end{array}$ & $\mathbf{F}$ & $\mathbf{P}$ \\
\hline Somatización & $0.39 \pm 0.5$ & $0.83 \pm 0.7^{\mathrm{a}}$ & $0.85 \pm 0.6^{\mathrm{b}}$ & $0.60 \pm 0.4$ & 7.47 & 0.000 \\
\hline $\begin{array}{c}\text { Obsesivo } \\
\text { Compulsivo }\end{array}$ & $0.97 \pm 0.7$ & $1.15 \pm 0.9$ & $1.11 \pm 0.8$ & $1.06 \pm 0.7$ & 0.49 & 0.687 \\
\hline $\begin{array}{c}\text { Sensibilidad } \\
\text { Interpersonal }\end{array}$ & $0.47 \pm 0.5$ & $1.00 \pm 0.9^{\mathrm{a}}$ & $0.85 \pm 0.7^{\mathrm{b}}$ & $0.96 \pm 0.8^{\mathrm{c}}$ & 5.82 & 0.001 \\
\hline Depresión & $0.53 \pm 0.5$ & $1.11 \pm 0.9^{\mathrm{a}}$ & $1.02 \pm 0.8^{\mathrm{b}}$ & $1.05 \pm 0.8^{\mathrm{c}}$ & 6.46 & 0.000 \\
\hline Ansiedad & $0.63 \pm 0.5$ & $1.07 \pm 0.8^{\mathrm{a}}$ & $1.03 \pm 0.7^{\mathrm{b}}$ & $1.01 \pm 0.7$ & 5.05 & 0.002 \\
\hline Hostilidad & $0.62 \pm 0.5$ & $0.81 \pm 0.7$ & $0.78 \pm 0.7$ & $1.05 \pm 0.8^{\mathrm{c}}$ & 2.89 & 0.036 \\
\hline $\begin{array}{c}\text { Ansiedad } \\
\text { Fóbica }\end{array}$ & $0.28 \pm 0.4$ & $0.60 \pm 0.6^{\mathrm{a}}$ & $0.52 \pm 0.5$ & $0.55 \pm 0.6$ & 3.73 & 0.012 \\
\hline $\begin{array}{c}\text { Ideación } \\
\text { Paranoide }\end{array}$ & $0.68 \pm 0.6$ & $1.13 \pm 0.7^{\mathrm{a}}$ & $1.10 \pm 0.8^{\mathrm{b}}$ & $1.29 \pm 0.7^{\mathrm{c}}$ & 6.28 & 0.000 \\
\hline Psicoticismo & $0.43 \pm 0.4$ & $0.87 \pm 0.7^{\mathrm{a}}$ & $0.74 \pm 0.6$ & $0.94 \pm 0.8^{\mathrm{C}}$ & 6.36 & 0.000 \\
\hline $\begin{array}{c}\text { Índice de } \\
\text { Severidad }\end{array}$ & $0.56 \pm 0.4$ & $0.93 \pm 0.6^{\mathrm{a}}$ & $0.89 \pm 0.5^{\mathrm{b}}$ & $0.94 \pm 0.5^{\mathrm{c}}$ & 6.49 & 0.000 \\
\hline $\begin{array}{c}\text { Índice de } \\
\text { Malestar }\end{array}$ & $1.47 \pm 0.4$ & $1.81 \pm 0.7^{\mathrm{a}}$ & $1.68 \pm 0.6$ & $1.71 \pm 0.5$ & 3.21 & 0.024 \\
\hline Total Síntomas & $19.01 \pm 10$ & $26.37 \pm 11^{\mathrm{a}}$ & $26.70 \pm 10^{\mathrm{b}}$ & $26.95 \pm 10^{\mathrm{c}}$ & 7.84 & 0.000 \\
\hline
\end{tabular}

Valores medios y desviación estándar de las dimensiones e índices del BSI. Comparación entre medias mediante análisis de varianza (ANOVA).

Comparación entre grupos mediante la prueba de Schefeé. Diferencias con $p<0.05$.

${ }^{a} \mathrm{G}$ Control vs G. Alcohol, ${ }^{\mathrm{b}} \mathrm{G}$ Control vs G. Heroína, ' $\mathrm{G}$ Control vs G. Cocaína.

No hay diferencias entre los Grupos Alcohol, Heroína y Cocaína.

cias estadísticamente significativas cuando se comparan los pacientes con el grupo de controles. Otros trabajos han encontrado la presencia de estos síntomas en diferentes muestras de pacientes dependientes a alcohol, heroína y cocaína.

En un estudio de Ochando y col (1999), los autores encuentran indicadores de desviación psicopática y componentes obsesivo-compulsivos en una muestra de pacientes alcohólicos. Algunos autores han señalado que la dependencia alcohólica podría considerarse como un trastorno en el control de impulsos, en el que el deseo de bebida sería equivalente a la ideación obsesiva y la conducta de consumo se correspondería con un comportamiento compulsivo (Rubio y Lopez, 1999). Modell y col (1990), también plantearon la existencia de un componente obsesivo-compulsivo en los sujetos alcohólicos. También aparecen trabajos en los que están presentes los síntomas depresivos en alcohólicos (Schukit, 1985; Van Hasselt, 1993).

También se ha detectado la presencia de síntomas psicopatológicos en dependientes a opiáceos. Reiger y col (1990) observaron en un estudio realizado con dependientes a opiáceos una mayor frecuencia de síntomas depresivos y ansiosos. Darke y col (1992) encontraron una estrecha relación entre niveles altos de psicopatología y un mayor consumo de heroína. En nuestro país, Saiz y col (1998) señalan la presencia de síntomas depresivos, somáticos, ansiosos y de disfunción social en una muestra de adictos a opiáceos.

Pueden encontrarse en la bibliografía estudios con pacientes dependientes a cocaína en los que se detecta sintomatología psicopatológica. En un trabajo pionero de Gawin y Kleber (1986) se señalaba que un 
$20 \%$ de las personas en tratamiento por adicción a cocaína cumplían criterios diagnósticos de trastorno del estado de ánimo. Gold (1993) señaló que las personas con sintomatología depresiva preferían utilizar estimulantes como la cocaína antes que otras drogas. Havassy y Arns (1998), encontraron que los pacientes con historia de abuso de cocaína presentaban síntomatologia psicótica y depresiva.

Respecto al grupo control se observa que las puntuaciones son globalmente bajas, pero resulta sorprendente la puntuación tan elevada en la dimensión obsesivo-compulsivo, en especial si dicha puntuación se compara con el resto de las dimensiones del mismo grupo. Creemos que ello puede ser debido a un sesgo de la muestra. Esta puntuación hace que no aparezcan diferencias significativas en esta dimensión cuando se compara el grupo control con el resto de grupos, aún cuando ésta dimensión es un indicador con puntuaciones muy elevadas en los grupos de pacientes.

Las puntuaciones en los índices globales (GSI, PSDI y PST) en todos los grupos de pacientes, pueden estar indicando un nivel e intensidad de distress psicológico importante que podría interferir en el mantenimiento de la abstinencia. Algunos autores (O'Connor y col, 1995) han señalado que las puntuaciones elevadas en los índices globales del BSI informan del miedo que experimentan los pacientes a recaer en el consumo de drogas y las dificultades que éstos perciben para mantenerse abstinentes.

Las comparaciones de las puntuaciones de todos los grupos nos han mostrado que no existen diferencias entre grupos de pacientes. Tales resultados podrían indicar que los distintos pacientes tienen en común la variedad de síntomas medidos por el instrumento de evaluación, confirmados por la información que aportan el índice de malestar, el índice de severidad y el total de síntomas. Por otro lado podría considerarse que el instrumento utilizado no sea sensible a la variedad de síntomas presentes en los distintos grupos, es decir, que no discrimine los efectos sobre la psicopatología de las diferentes sustancias, sino que sólo aporte información sobre el nivel de malestar experimentado por los pacientes. Es posible que las características psicométricas del instrumento sólo nos permitan detectar la existencia de un conjunto de síntomas inespecíficos y sin predominios claros entre los distintos pacientes.

Otro aspecto a considerar son las diferencias encontradas en las características sociodemográficas de los pacientes, en especial las diferencias del grupo alcohol con el resto de pacientes. Es posible que la mayor edad de estos pacientes y el mayor número de años de consumo influya de alguna forma en los indicadores de psicopatología. La posible asociación entre este tipo de variables debería tenerse en cuenta en posteriores estudios.
Detectar la psicopatología en los pacientes dependientes a drogas es una tarea importante en el abordaje terapéutico de estos pacientes. La gravedad psicopatológica presenta una relación inversa con la evolución positiva en el tratamiento (McLellan y col, 1983; O’Brien y col, 1984). La evaluación de la psicopatología y la utilización de instrumentos de screening, es una tarea fundamental en la planificación individualizada de los tratamientos del abuso de drogas. La utilización de un instrumento como el BSI debe de ser contrastada con más estudios en los que se utilicen muestras más homogéneas y con un número más elevado de pacientes.

\section{REFERENCIAS BIBLIOGRÁFICAS}

Abbot, P ; Weller, S; Walker,S (1994): "Psychiatric disorders of opioid addicts entering treatment: preliminary data". Journal of Addictive Diseasses, Vol 13(3): 1-11.

Asociación Psiquiátrica Americana (1995): "Manual diagnóstico y estadístico de los trastornos mentales". Ed Masson. Barcelona .

Blaszczynski, A; Steel, Z; McConaghy, N: (1997): "Impulsivity in pathological gambling : the antisocial impulsivity ". Addiction . Vol. 92 (1): 75-87

Calsyn, D; Fleming, CH; Wells, E; Saxon, A. (1996): "Personality Disorders subtypes among opiate addicts in methadone maintenance". Psychology of addictive behaviors. Vol 10 (1):3-8.

Catton, C. Gralnick, A. Bender, S.(1989): "Young chronic patients and substance abuse". Hos. Community Psychiatry, 40: 1037-1040.

Cuadrado, P. (1998): "Evolución de la dependencia alcohólica en tratamiento. Fractores predictivos en un seguimiento de 5 a 7 años". Adicciones, vol 10 n 4: 335-341.

Darke, S. Wodak, A. Hall, W. Heathern, N. Ward, J. (1992). Prevalence and predictors of psychopatology among opioid users. British Journal of Addictions.

De Vicente P. Sánchez, N. Ochando, B (1999): "Prevalencia de trastornos de personalidad en pacientes con dependencia alcohólica". Trastornos Adictivos, vol 1, n³-4. Comunicación presenctada en el VII Congreso Nacional de la Sociedad Española de Toxicomanías.

Derogatis, L. y Cleary, P. (1977): "Confirmation of the dimensional structure of the SCL-90. A study in construct validation". J. Clin. Psychology 33(4): 981-989.

Derogatis, L. (1975): "Brief Symptom Inventory". Baltimore. Clinical Psychometric Research.

Derogatis, L.(1983): "SCL-90-R. Administration, scoring and procedures manual". Clinical Psycometric Research.

Fielman, N; Woolkolf, R, Allen, L.(1995): "Dimensions of self-concept : a comparison of heroin and cocaine addicts ". American Journal of drug and alcohol abuse. Vol 21 (3): 315-326.

Gawin, F. y Kleber R, H.: "Abstinence symptomatology and psychiatric diagnosis in cocaine abusers: clinical observations". Arh. Gen. Psychiatry, 43: 107-113.1986 
Gold, M.(1993): "Manifestaciones clínicas de la cocaína". En: Cocaíne. Plenum Press. New York. Ed en Neurociencias.

Gold, M (1989): "The good news about panic, ansiety and phobias". New York. Villar Books.

Gutierrez, E; Saiz, P; Gonzalez, P; Fernandez, J; Bobes, J: (1998): "Trastornos de personalidad en adictos a opiáceos en tratamiento con agonistas vs antagonistas". Adicciones, 10 (2): 121-129.

Havassy, B y Arns, P. (1998).:"Relationship of cocaine and other substance dependece to well-being of high risk psychiatric patients". Psychiatric Services, vol 49(7): 935-940.

Jellinek, E.M. (1960): "The disease concept of alcoholism". New Haven CT, College and University Press.

Khantzian, E. J; Treece, C. J. (1985): "DSM-III psychiatric diagnosis of narcotic addicts: Recent findings". Archives of General Psychiatry. Vol 142: 1067- 1071.

McLlellan, A. Luborsky L, Woody, O’Brien, C. Druley, J (1983). Predicting response to alcohol and drug abuse treatment: role of psychiatric severity. Arch. Gen Psychiatry, 40: 620-25.

Miller, N (1996): " Issues in the diagnosis and treatment of comorbid addictive and other psychiatric disorders". Addiction psychiatry : Current diagnosis and treatment New York

Miller, N. (1995): "Addiction psychiatry: current diagnosis and treatment". Willy-Liss. New York.

Modell, J.G. Mountz, J.M. Beresdoft, T. (1990): "Basal ganglia/limbic striatal and thalamocortical involvement in craving and loss control in alcoholism". J. Neuropsychiatry Cli. Neurosciencie 2: 123-144.

Neff, J. A. (1993): "Life stressors, drinking patterns, and depressie symptomatology: ethniticy and stress-buffer effects of alcohol". Addictive Behavior, vol 18: 373-387.

Nunes, E. Quikin, F. Rosecan, J.(1986): "Psychiatric diagnosis in cocaine abuse: evidence for biological heterogeneity". Pressented at the British Association for Psychopharmacology, Cambridge, England, July 14.

O’Brien, C. Woody, G. McLlelan, A.(1984) Psychiatric disorders in opioid dependent patients. J Clinic Psychiatry, 45: 9-13.

O'Connor LE, Berry JW, Morrison A, Brown S. (1995): The drug of choice phenomenon psychological differences among drug users who preferred different drugs. Int J Addict Apr;30(5): 541-55.

Ochando, B. De Vicente, P. Valderrama, J. (1999): "Estudio de la adhesión a tratamiento de pacientes con síndrome de dependencia alcohólica". Trastornos Adictivos, vol 1, $n^{\circ}$ 3-4. Comunicación presentada en el VII Congreso Nacional de la Sociedad Española de Toxicomanías.

Post, R. Weiss, S. Port, A.(1992): "Sensitization and kindling effects of chronic cocaine administration". En
Lakowsky, Galloway y Ehite (ed): Cocaine: pharmacology, physyology and clinical strategies. Boca Raton FL, CRC Press.

Reiger, D. Farmer, M Rae D. Locke, B. (1990). Comorbidity of mental disorders with alcohol and other drug abuse: Results from the Epidemiologic Catchment Area. J. A. Medical Association, 264: 2511-2518.

Richman, S. A; Tichman, M; Fine, E. W. (1980): "Psychosocial differences between male and female alcoholics". En: Glanter M, ed. Currents in alcoholism. New York.

Rousanville,B. J ; Tierney, T. ; Crits-Christoph., K; (1982): "Predictors of treatment outcome in opiate addicts: Evidence for the multidimentsionality of addicts problems". Comprehensive Psychiatry. Vol.23: 462478.

Rubio, G. Lopez, M. (1999): "Validación del cuestionario sobre los componentes obsesivo- compulsivo de bebida en alcohólicos españoles". Adicciones, vol 11, $n^{\circ} 1$ : 7-15.

Saiz, F. Ruiz, I. Salvador, L (1998). Estudio de detección de problemas psiquiátricos en una muestra de pacientes dependientes de opiáceos en tratamiento. Rev. Esp. Drogodependencias, 23(2): 161-168.

Ssn Narciso, G; Carreño, J; Pérez, S; Alvarez, C; Gonzqlez, M; Bobes, J: (1998): "Evolución de los trastornos de personalidad evaluados mediante el IPD en una muestra de pacientes heroinómanos en tratamiento con naltrexona". Adicciones, 10 (1): 7-21

Sánchez-Hervás, E. Tomás, V. Climent, A (1999): Trastornos de personalidad en adictos a opiáceos. Adicciones 1999, $11(3): 221-227$

Sayette, M. (1993): The effect of alcohol on emotional response to a social stressor. J Studies on Alcohol, vol 53 (6): 345-352

Schukit, M. (1985): "The clinical implications of primary diagnostic goups among alcoholism". Arch. Gen Psychiatry, 137: 1043-1049.

Siegel, R (1982).: "Cocaine smoking". J Psychoactive Drugs 14:271-359.

Sorel, E.(1990): "Cocaína, depresión y familia". Psicopatología, vol 10, n4: 192-194.

Van Hasselt, V. B. (1993): "Social skills and depression in adolescent substance abusers". Addictive Behavior, vol 18: 9-18.

Wojnar, M; Wasilwski, D ; Matsumoto, H ; Cedro, A. (1997): "Differences in the source of alcohol withdrawal in women and men: a Polis sample". Alcoholism: Clinical and Experimental Research. Vol 21 (8): 1354-1355.

Yates, W; Fulton, A; Gabel, J (1995): "Factores de riesgo de la personalidad del consumo de cocaína". Rev. Esp. Toxicomanías, n³: 3-5. 